\title{
An experimental study on shear bands in sand using the orthogonal cutting setup
}

\author{
Abhijit Hegde ${ }^{1, *}$ and Tejas Murthy ${ }^{1, * *}$ \\ ${ }^{1}$ Department of Civil Engineering, Indian Institute of Science, Bangalore 560 012, India
}

\begin{abstract}
We study the evolution of shear bands in granular media subjected to very large deformations using the orthogonal cutting geometry. We perform our cutting experiments on Cauvery Delta sand with $d_{50}$ of $0.45 \mathrm{~mm}$. We also capture images of the cutting process which allows us to perform a PIV analysis to determine the deformation in material around the cutting tool. In our experiments we observe that the dynamic angle of repose of the pile that forms in-front of the tool as cutting progresses, approaches the critical state friction angle of the material. We observe a flow bifurcation around the tool as the material begins to slip along planes which are oriented at an angle of $\pi / 4-\theta / 2, \theta$ being the dynamic angle of repose, to the cutting direction. This leads to formation of shear bands/velocity jumps which contain around 14 particles. A measurement of dilation angle within the shear bands indicate that material is in a critical state of deformation.
\end{abstract}

\section{Introduction}

Shear bands are ubiquitous and form in all materials including granular media. They make the overall deformation field inhomogeneous. From an engineering perspective, they are undesirable as they can lead to energy loss in food processing industries, grain storing silos,etc., and are also responsible for catastrophic events such as avalanches and failure of embankments.

Hence shear bands warrant a detailed study, both qualitatively and quantitatively. It becomes imperative that for better design of engineering equipment that handle granular materials, it is necessary to study the properties of shear bands and their overall relation to both external boundary conditions as well as internal constituents of the ensemble.

In-case of shear bands that form in granular media, they are studied in two distinct classes, ones that form near the boundaries [1,2] and ones that form within the bulk of the material [3]. We present experiments on the orthogonal cutting geometry, wherein we are able to generate steady shear bands within the bulk of granular material, whose properties seem to remain more or less the same throughout the experiment. Hence, the set-up provides us with a window into the phenomenon of shear banding in granular materials, not just in the bulk, but also at the boundaries(appearing along the tool-material interface) allowing us to study their behavior in detail over long time intervals. In this letter, we investigate properties of shear bands which form inside the bulk of the material.

The problem of trenching/ploughing, is of critical importance to the civil engineering community [4] and is the main motive for our current study. This problem can be

\footnotetext{
*e-mail: abhijithegde@iisc.ac.in

**e-mail: tejas@iisc.ac.in

A video is available at https://doi.org/10.48448/emzd-ph40
}

modeled as a plane strain, orthogonal cutting problem, in a similar vein to machining in metals [5].

\section{Experimental set-up}

The experimental set-up employed by us to study the problem of orthogonal cutting of granular materials(sand) is shown in Fig. 1. The set-up consists of an industrial grade Aluminium box of size $555 \mathrm{~mm} \times 160 \mathrm{~mm} \times 15 \mathrm{~mm}$ with the front face and top faces removed. Slots were cut onto the bottom and side panels of the box to place a transparent glass plate for imaging the cutting process. Sand was filled into the box using a funnel(air pluviation method) resulting in a packing fraction of around 0.6. The box was then mounted onto a CNC machine(Ace Micromatic Pvt.Ltd). A tool made of High-speed steel was fit into the spindle of $\mathrm{CNC}$ machine and lowered into the box through the space between the box and the glass plate. The box is then translated at a constant velocity to perform our experiments. Images of the cutting process were captured around the stationary tool using a high speed camera(PCOHS1200), which was placed perpendicular to the front face of the box, at a frame rate prescribed by the cutting speed and with proper illumination of the region around the tool. The resolution of the images captured during the process was $5.3 \mathrm{pix} / \mathrm{mm}$. The images were then analyzed using a PIV code written in MATLAB. PIV is an indirect, non-intrusive, whole field velocity measurement technique, which can be used efficiently to obtain the deformation field. We have used a non-overlapping correlation window size of 40pix x 40pix and a maximum error not exceeding 3\% was observed while measuring the velocities. 


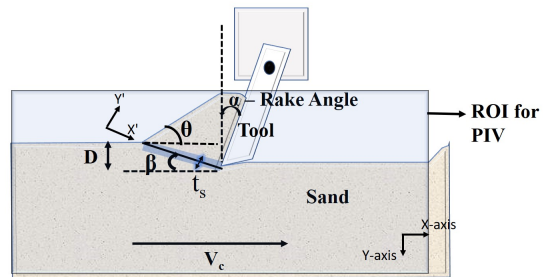

Figure 1. Schematic of the experimental set-up with D being the initial depth of cut and $V_{c}$, the cutting speed

\section{Materials used}

In this paper, we have performed experiments on Cauvery Delta sand with a $d_{50}$ of $0.45 \mathrm{~mm}$. The physical as well as mechanical properties of this granular material has been studied extensively by [6]. The sand grains are angular with a roundness $(\mathrm{R})$ value of 0.17 .

\section{Results}

The main parameters that have been often reported in orthogonal cutting experiments are the rake angle $(\alpha)$, cutting velocity $\left(V_{c}\right)$ and the initial depth of $\operatorname{cut}(\mathrm{D})$ [7]. Our experiments are performed at a rake angle of $0^{0}$ and a cutting speed of $10 \mathrm{~mm} / \mathrm{min}$. The initial depth of cut is $20 \mathrm{~mm}$. Hence, the imposed velocity provides us with a natural strain rate scale given by $V_{c} / D\left(8.35 * 10^{-3} \mathrm{~s}^{-1}\right)$, and has been used to normalize our strain rate results. The dimensions of the set-up have been normalized by representative particle size, $d_{50}$ and the tool displacement by initial tool depth, D.

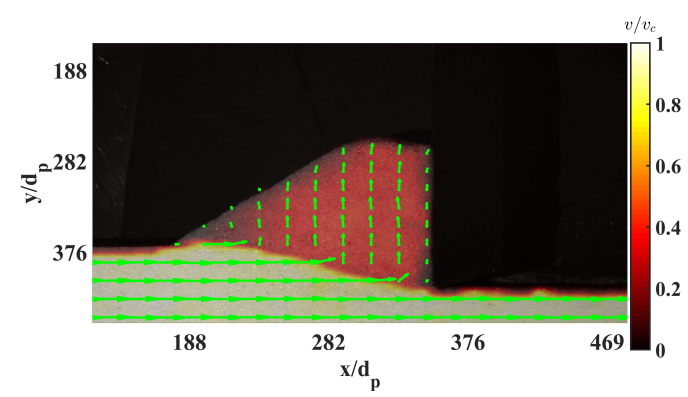

Figure 2. Velocity field obtained from PIV. The present image corresponds to a time instant when the tool has moved by a distance of $5^{*} \mathrm{D}, \mathrm{D}$ being the initial depth of cut

In Fig. 2, we present the velocity field obtained from our PIV analysis. We have superposed a quiver plot on top of a contour map indicating the velocity magnitude, in-order to visualise the flow. The particles above the tool tip undergo a sharp change in their direction as they approach the tool while particles below continue to move in the same direction unimpeded, and this results in a flow bifurcation. In the bifurcation region, the particles roll over one another along planes with a definite orientation, before being pushed upward along the rake face and undergo significant retardation with the velocity magnitude reducing by $50 \%$. As more of the material gets cut,the particles moving upwards begin to repose under their own weight. The free surface angle reaches a steady value with continued cutting. The overall pattern of the velocity field does not change much with the progress of cutting, in the sense that it is self-similar, with just the size of the pile changing.

The strain rate was computed from the velocity field using the equation

$\begin{aligned} \dot{\boldsymbol{\epsilon}}_{e f f}= & {[4 / 9} \\ & \left.*\left(1 / 2 *\left[\left(\dot{\epsilon}_{x x}-\dot{\epsilon}_{y y}\right)^{2}+\left(\dot{\epsilon}_{x x}\right)^{2}+\left(\dot{\epsilon}_{y y}\right)^{2}\right]+3 / 4 * \dot{\gamma}_{x y}^{2}\right)\right]^{1 / 2}\end{aligned}$

$\dot{\epsilon}_{x x}=\delta u / \delta x$ being the normal component of strain rate tensor in the direction of tool traverse and $\mathrm{u}$, the horizontal displacement.

$\dot{\epsilon}_{y y}=\delta v / \delta y$ being the normal component of strain rate tensor in the direction perpendicular to tool traverse, $\mathrm{v}$ being the vertical displacement.

$\dot{\gamma}_{x y}=1 / 2 *[\delta u / \delta y+\delta v / \delta x]$ being the shear component of strain rate.

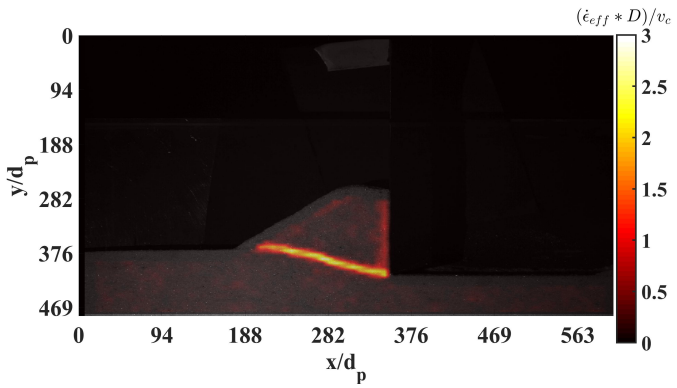

Figure 3. Strain rate field obtained from the velocity field. A localized region of intense strain rate is clearly visible in the figure

In Fig. 3, we have presented the strain rate field corresponding to the velocity field shown in Fig. 2. In the strain rate field we clearly observe formation of a narrow region of intense strain rate (3 times the imposed strain rate) centered around the tool tip and reaching the free surface, along which the material gets cut. The region is localized spatially and it makes a well defined angle with respect to the horizontal(direction of cutting). These narrow regions of strain localization are termed as shear bands and they cause the deformation field to become inhomogeneous, rendering boundary measurements of strain meaningless. We also observed regions of high strain rate at the tool-sand interface and near the free surface of the pile.

The angle of repose of the pile is plotted in Fig. 4. The steady value of repose angle is close to $42^{0}$. By comparing with the hollow cylinder experiments [6] carried out for a ' $b$ '(intermediate principal stress ratio) value of 0.4 (which corresponds to plane strain conditions) on the sand 
used in our experiments, we observe that the repose angle of the pile is very close to the critical state friction angle obtained using the hollow cylinder tests under similar boundary conditions.

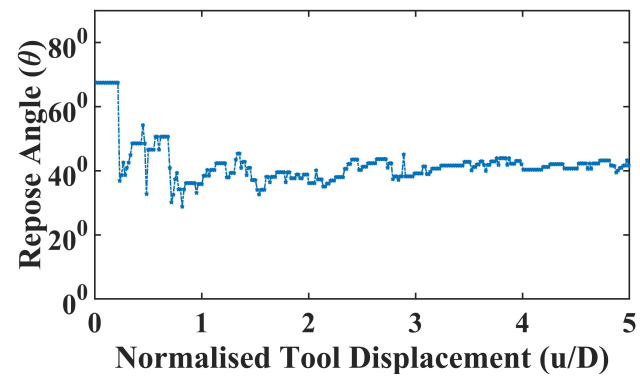

Figure 4. Evolution of repose angle as a function of tool displacement

\subsection{Determination of shear band inclination angle}

In-order to determine the inclination angle of the shear band, we have used the Standard Hough Transformation technique, which has been used to identify lines in an image. More information regarding the technique can be obtained in [8]. The Hough transformation has been performed on the strain rate contour field (Fig. 3) in this study. Similarly, the velocity field can also be used to the same effect.

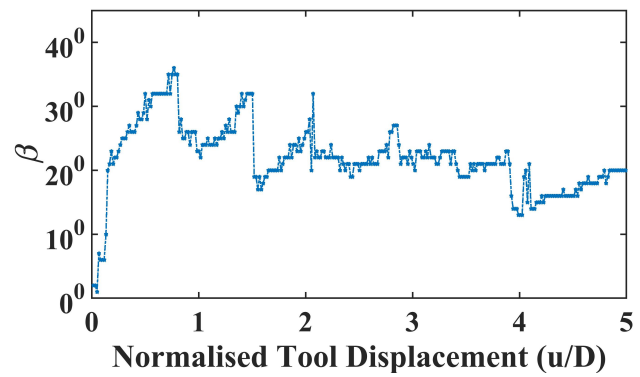

Figure 5. Evolution of inclination angle of shear band, $\beta$, measured with respect to the direction of cutting

The angle determined during each frame has been collated and presented in Fig. 5. The angle of inclination is higher during the initial stages of cutting (maximum value measured is close to $36^{0}$ ), which we conjecture is mostly due to its proximity to the tool, as we often observe shear bands forming at the interface between the tool and the pile. The angle tends to increase and decrease periodically similar to observations made by [9], with the mean value, averaged over the last 150 frames, being $22^{0} \pm 2^{0}$. We notice that this angle is close to $\pi / 4-\theta / 2, \theta$ being the angle of repose of the pile measured in our experiments (Fig. 4). It is well known from Mohr-Coulomb theory that granular materials fail along planes which are inclined at an angle of $\pi / 4-\phi / 2, \phi$ being the internal friction angle, to the direction of major principal stress (which happens to be the direction of cutting in our case [10]). These planes have been termed as Coulomb rupture planes. Our experimental results suggest that shear bands align themselves along the Coulomb planes if we were to replace $\phi$ by dynamic angle of repose $\theta$, which, as mentioned earlier, corresponds to the critical state friction angle of the material. Therefore, the dynamic repose angle of granular materials indeed corresponds to its internal friction angle at critical state and remains to be verified under different geometries.

\subsection{Measuring the thickness of shear bands}

The thickness of shear bands is of critical importance to any continuum theory trying to model the mechanical behavior of granular materials. It provides an intermediate length scale, between the particle level of interactions and the macroscopic deformations measured. Also shear bands correspond to regions across which granular materials undergo phase transition from a solid to fluid like state. Though many experiments have been conducted, typically in a biaxial or triaxial apparatus, to determine the final thickness of such shear bands, it is not possible to study the characteristics of these critical localization phenomena under very large deformations using such classical approaches. Orthogonal cutting geometry is especially suitable under such circumstances. It allows the researcher to generate shear bands whose properties remain more or less steady throughout the entire cutting process and makes possible, to perform a detailed study of these interesting features in granular materials.

In-order to determine the thickness of the shear bands formed during the cutting process, we have used the data on inclination angle from our previous analysis. A line is drawn perpendicular to the shear band as shown in Fig. 6 and the strain rate profile along the line is determined. Similar lines are considered along the length of the shear band, and the spatial average is considered. A typical profile of shear strain along such a line (taken midway along the length of shear band) is shown in Fig. 7. We observe that the profile is Gaussian. We have used the concept of FWHM (Full Width at half maximum) to determine the extent of localization using the strain rate profile. Evolution of the thickness of shear band as cutting progresses, is shown in Fig. 8. We observe that the thickness fluctuates in time. Identifying the region of localization within the image also allowed us to make a measurement of dilation angle within the shear band. As in the case of thickness of the shear band, strong fluctuations were also observed in the measured values of dilation angle (Fig. 9). The mean value (averaged in time) of shear band thickness was $14 \pm 2 * d_{p}$, which is only slightly less than the theoretically predicted value of $16 * d_{p}$ by [11] based on bifurcation analysis. The evolution of dilation angle with tool displacement is shown in Fig. 9. The average dilation angle within the shear band is close to $1^{0}$, hence clearly indicating that the material has a reached a critical state of deformation, making the plastic flow within the shear band non-associative.

In fact the shear bands obtained in our PIV analysis should correspond to velocity discontinuities [12] and hence are supposed to be inclined at angle of $\pi / 4-\psi / 2$ ( $\psi$ being the dilation angle) to the direction of major principal strain rate. This implies that the direction of major 
principal strain rate does not coincide with the direction of major principal stress. The direction of major principal strain rate(which is compressive) is inclined at angle of $-22^{0}$ to the $\mathrm{x}$-axis, as the tool pushes into the material in-order to dislodge it from its position.

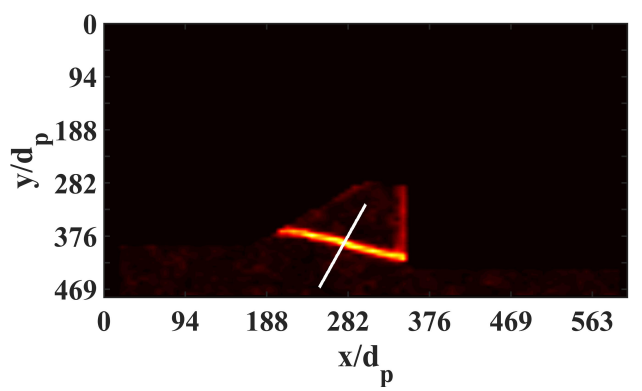

Figure 6. Sample line along which the strain rate profile has been measured in our experiments

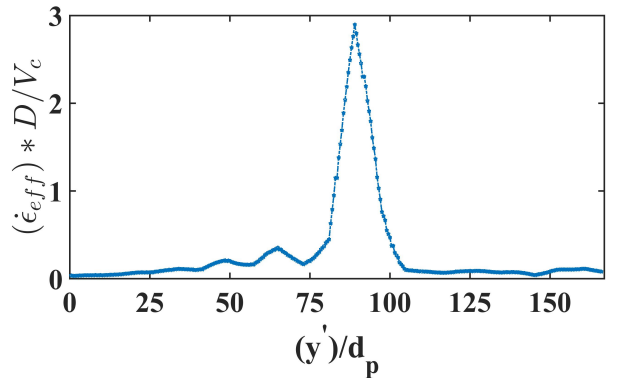

Figure 7. Typical strain rate profile measured along lines perpendicular to the shear band

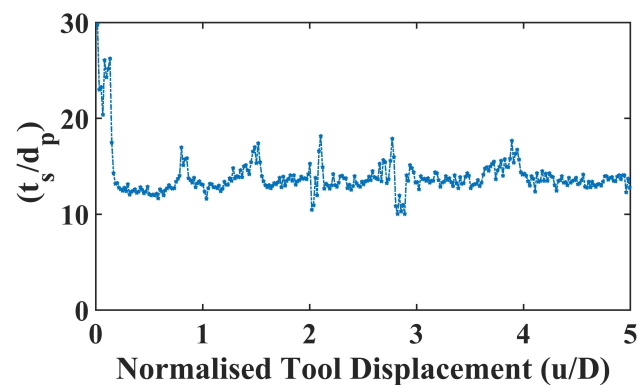

Figure 8. Evolution of shear band thickness, $t_{s}$ as a function of tool displacement

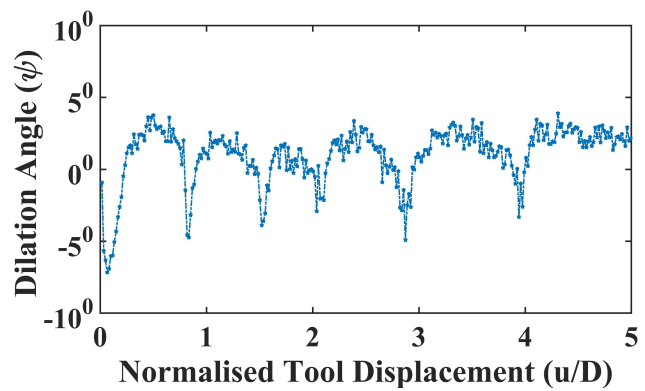

Figure 9. Evolution of dilation angle within the shear band as a function of tool displacement.

\section{Conclusion}

We use orthogonal cutting set-up to quantitatively study large deformations in granular materials. We generate consistent shear bands within the system and their properties can be very easily studied using simple imaging techniques like PIV. In our work, using this orthogonal cutting geometry, we study the overall deformation field as well as the behavior of shear bands forming within the bulk of Cauvery Delta sand with a $d_{50}$ of $0.45 \mathrm{~mm}$, both qualitatively and quantitatively. We observe that as the cutting progressed, material removed from the bulk formed a pile in-front of the tool and the free surface angle of this pile was found to be very close to the critical state friction angle of the granular material. The measured thickness of the shear bands was close to the value predicted by the bifurcation theory and the orientation of the shear band seems to coincide with the Coulomb rupture planes. The material within the shear band is in a critical state of deformation. We believe that these experimental observations from orthogonal cutting geometry could be used to benchmark DEM simulations.

\section{References}

[1] D.M. Mueth, G.F. Debregeas, G.S. Karczmar, P.J. Eng, S.R. Nagel, H.M. Jaeger, Nature 406, 385 (2000)

[2] R. Nedderman, C. Laohakul, Powder Technol 25, 91 (1980)

[3] D. Fenistein, J.W. van de Meent, M. van Hecke, Phys Rev Lett 92, 094301 (2004)

[4] J.P. Hambleton, S. Stanier, D.J. White, S.W. Sloan, Aust Geomech J 49, 147 (2014)

[5] P. Dewhurst, I. Collins, Int J Numer Meth Eng 7, 357 (1973)

[6] R.K. Kandasami, T.G. Murthy, Granul Matter 19, 21 (2017)

[7] E. McKyes, Soil cutting and tillage (Elsevier, 1985)

[8] R.O. Duda, P.E. Hart, Commun ACM 15, 11 (1972)

[9] N. Gravish, P.B. Umbanhowar, D.I. Goldman, Phys Rev Lett 105, 128301 (2010)

[10] Y. Hatamura, K. Chijiiwa, B JSME 18, 619 (1975)

[11] H.B. Mühlhaus, I. Vardoulakis, Geotechnique 37, 271 (1987)

[12] K.H. Roscoe, Geotechnique 20, 129 (1970) 